\title{
Review on the Development and Evolution of Foreign Sports Competitive Intelligence
}

\author{
Wen Yue \\ Department of Physical Education, Beihang University, Beijing, China \\ Email address: \\ sqxs9@buaa.edu.cn \\ To cite this article: \\ Wen Yue. Review on the Development and Evolution of Foreign Sports Competitive Intelligence. Social Sciences. \\ Vol. 7, No. 5, 2018, pp. 216-222. doi: 10.11648/j.ss.20180705.13
}

Received: August 21, 2018; Accepted: September 4, 2018; Published: October 13, 2018

\begin{abstract}
Using the research methods of historical analysis, expert interview, mathematical statistics and so on, the present research has, based on the review of sports competitive intelligence development in foreign countries, divided into in three evolution stages according to the features in development, namely, pioneer stage, traditional sport intelligence stage, and modern sport intelligence stage. By analyzing the achievements in each stage, it has proposed the developmental trends in future sport competitive intelligence. Along with the rapid development of modern information technology like the Internet, cloud computing, big data, the development of sport competitive intelligence exhibits the trends of diversified service objects, hi-tech methods and means, multidimensional research paradigm, etc. The prospect of sport competitive intelligence can be summarized as the following: continued development of technology will bring more high- tech data acquisition software and equipment to the field; new multivariate statistical model will make sports competitive intelligence work more forward- looking and predictive.
\end{abstract}

Keywords: Foreign, Sports Competitive Intelligence, Evolution, Review

\section{Introduction}

Sports intelligence is the dissemination of new sports knowledge with specific needs, including useful new knowledge in sports management, teaching, scientific research and training competitions, etc [1]. Foreign sports intelligence work began in the late 1940s and early 1950 s. Competitive intelligence, produced in the 1950s and rising in the 1980s, provided the conditions for the evolution of sports intelligence. As a result, sports competitive intelligence gradually rose. Sports competitive intelligence refers to the intelligence research on competitors, competitive environment and own information conducted by the competitive subject to gain competitive advantage in sports management, teaching and training competition and other aspects, which provides strategic analysis information for decision-making level. It is a significant development of sports intelligence. So that sports intelligence has a way out of the predicament. This research uses the historical analysis method, according to the foreign sports competitive intelligence development characteristics in the time dimension presents the difference, carries on the stage division to its development process. After listening to the experts to verify the reasonableness of this division, the last of the three important stages of foreign sports competitive intelligence evolution were obtained by integrating the experts' opinions.

\section{Pioneer Stage: Before the Concept of Sports Intelligence Was Clearly Proposed (From the End of 1940s to the Middle of 70s)}

\subsection{The Background of the Generation of Sports Intelligence Work}

Foreign sports intelligence work began in the late 1940s and early 1950s. After the end of the second world war, all countries were liberated from the war mentality and began to devote themselves to the improvement of social material life and people's cultural quality. In such an environment, sport is booming, people's awareness of sport is gradually awoken, and sports communication between countries is increasingly frequent. Sport has become an integral part of the construction of social culture and spiritual civilization. With the increasing importance of sports in social life, the number of sports 
literature has increased rapidly. People's attention to sports urges them to acquire the useful new knowledge of sports accurately, timely and effectively, but this need cannot be satisfied under the technical conditions of sports itself. At this time, under the continuous progress of science and technology, scientific intelligence can be widely used in other disciplines. Therefore, people apply it to the processing of sports literature with the help of rapidly developing scientific intelligence, resulting in systematic and organized sports literature processing.

\subsection{The Primary Form of Sports Intelligence Work -- Sports Literature Work}

In 1948, sports intelligence work was born in the form of sports bibliographies and abstracts. The sports intelligence work was first completed by the new paper compilation and transmission method in the sports literature processing under the leadership of professor Josef Recla, dean of the school of physical education at the university of graz in Austria, and obtained the international related domain experts spoke highly of and widely recognized. These methods are: 1) classification, catalogue compilation and abstract processing of the papers; 2) convening academic meetings, papers submitted according to the theme of the meeting; 3) organizing exhibitions of sports papers.

In view of the significant role of sports intelligence in understanding the development trend of international sports, enriching knowledge and improving work efficiency, etc., countries attach more and more importance to it. Therefore, the institutions that systematically carry out sports intelligence work have emerged in each country, and each institution has carried out paper compilation in its own way. For example, in 1955, the German University of Physical Culture's card-type sports literature search tool, and in 1958, the world's first sports digest publication, analysis review, led by Belgian scholar Julien Falize. These successive mechanisms can be roughly divided into two types. One is to establish sports intelligence organizations scattered throughout the country, forming a "multi-center" situation, which is mainly built by the libraries of sports colleges and universities according to their needs, such as the United States, Japan, and most capitalist countries and developing countries belong to this type. The other is the establishment of the country's only sports information center, to form a centralized research and management model, such as the Soviet union, west Germany, Cuba and eastern European countries. However, no matter the above types, their main task is to collect and compile physical literature and disseminate them in the form of abstracts and periodicals. Taking Cuba as an example, the sports intelligence center of the country has more than 80 employees, whose work is mainly to collect sports intelligence around the world, such as scientific research trends, training plans, sports performance and records, etc., and compile the information and provide it to users in various forms. The main way of collection is to establish extensive international communication, for which three kinds of sports publications are published for international exchange. On the basis of the collection of a large number of international sports publications, the compilation of information archives of national elite athletes, statistics of the technical progress of elite athletes and so on.

\subsection{The Establishment of the International Association for Sports Intelligence (IASI)}

With the expansion of sports literature exchange and demand, the voice of establishing international sports intelligence alliance is getting higher and higher. Therefore, professor Josef Recla, professor Julien Falize and Dr. Walther Arnold, library director of German university of physical culture launched the first international sports literature intelligence organization, the international sports literature intelligence agency, during the 1960 Rome olympic games. The international sports literature intelligence agency is part of the united nations educational, scientific, and cultural organization, chaired by Walter Arnold. Since then, the international sports literature intelligence agency has held a series of seminars, knowledge lectures, professional training, and so on, and deeply discussed new ideas and methods of sports literature intelligence work. The content covers the development and use of retrieval tools, the compilation of literature, and the automatic intelligence processing technology, which greatly promotes the rapid development of sports literature intelligence in the world.

In view of the rapid development of international sports intelligence work, under the leadership of president Walter Arnold, the international sports literature intelligence agency changed its name to "International Association for Sports Intelligence" at the expansion meeting of the international sports literature intelligence agency's executive committee in 1974. The international association for sports intelligence has eight committees. They are sports glossary, noun terms, classification, information, information means, literature data, sports library and information personnel training. More than 170 individual members and a handful of collective members from more than 30 countries have joined the group. Since then, international sports intelligence academic exchange activities have been carried out more widely, international sports intelligence work has rapidly expanded to multilateral cooperation.

To sum up, the main achievements and characteristics of the pioneer stage are as follows. 1) In Austria, east Germany and Belgium, sports intelligence appeared in the form of catalogue and abstract of sports books and then became an integral part of sports practice. 2) Sports intelligence centers have been set up successively in various countries, some of which are derived from the libraries of sports colleges, some are built by government agencies or individual sports associations according to the needs. The main task is to collect and compile sports literature materials and disseminate them in the form of publishing abstracts and reviewing periodicals. 3) The establishment of international association for sports intelligence has promoted the internationalization development of sports intelligence work. 4) The sports intelligence work has gradually become scientific and has 
made progress in audio-visual technology and automatic intelligence processing. Although these achievements were achieved in the pioneer stage, the sports intelligence theory in this stage is neither systematic nor complete, and the level of practical application is not high, which can be seen as the accumulation stage that must be experienced.

\section{Traditional Sports Intelligence Stage (From the End of 1970s to the Middle of 90s)}

\subsection{The Proposed of the Concept of Sports Intelligence}

Since the 1970s, sports intelligence workers have accelerated the development of sports intelligence with the help of modern scientific and technological means such as rapidly developing computer technology and telecommunication technology. In 1977, professor Josef Recla, dean of the school of physical education, university of graz, Austria, proposed at the sixth congress of the international association for sports intelligence: "sports intelligence science refers to the science of in-depth research and the provision of creative sports intelligence, which belongs to sports science. Specifically, it is the upper limit concept of all work in the sports literature (such as literature, data literature, information carrier literature, audio-visual literature, etc.) and sports intelligence. The emergence of the concept of sports intelligence indicates that the sports intelligence work begins to rise to a certain theoretical level. In 1985, Olsen, a professor at the Norwegian university of sport and physical education who was then president of the international association for sports intelligence in the international association for sports intelligence eighth congress and the second world sports intelligence congress pointed out that: "Although the concept of sports intelligence science proposed by professor Josef Recla in 1977 has not been widely accepted so far, the main basis for the future of sports intelligence science is that if sports science can have an impact on sports practice, it must be because the scientific research results are influential. Therefore, the intelligence research and its effect on sports will be as important as other factors that affect sports performance, perhaps as important as the research itself." [2]

However, some scholars hold a negative attitude towards the concept of sports intelligence science, such as Japanese scholar professor Takao Fujito (1986) in the "Japanese Sports Intelligence Work" mentioned that sports intelligence has not yet reached the "science" level, he pointed out: 1) If sports intelligence develops into "science" must have its own research areas and research methods; 2) The scientific research achievements of sports intelligence science have not reached the ability to discover new things and reveal the laws of new things. At present, the present achievements belong to the category of intelligence science and library science. For example, the German scholar Professor Gail Ebner and Professor Kitts (1988) in the "The Relationship Between Scientific Intelligence and Sports Science" mentioned:
"Scientific intelligence is scientific knowledge which is brought into the process of social communication by means of material carrier. Sports intelligence can be classified as scientific intelligence. [3]. This shows that although many experts and scholars have made great efforts to construct their own theoretical system of sports intelligence science, but in the view of other scholars, the concept of sports intelligence science can not withstand deliberation.

\subsection{The Rise of Sports Information Research}

In the absence of unanimous recognition of sports intelligence science in the academic circle, the vigorous development of the knowledge economy characterized by informatization in the late 1980 s led to a debate between "information" and "intelligence", which pushed intelligence science into a dilemma. Some scholars even used "a historical misunderstanding" to describe the establishment of intelligence science. Therefore, there was a great debate in the academic circle on the issue of the survival of intelligence science, namely the famous "debate of the century." At the same time, under the influence of the intelligence community, the sports intelligence also encountered the survival crisis.

During this period, several congresses of the international association for sports intelligence focused on discussing various issues of sports information and sports intelligence. Among them, professor Feilinbofu (1988) wrote in his article "Several Theoretical Problems of Information Assurance in the Field of Sports" after the conference: "Some theoretical problems of information assurance should be expounded from the perspective of sports information which is broader than sports intelligence concept [4]." In the article "Problems and Prospects of Sports Informatization”, Nepal Ponomarev (1995) of St. Petersburg state institute of physical education pointed out: the most important problem of sports informatization is to develop sports science which can provide new knowledge. The information people get should not only be stored in libraries, information department libraries and laboratory shelves, but also be transformed into information products that are easy to understand and master by every expert, every citizen and foreign demander [5]. This is similar to what professor Olsen said in his 1986 article "The Development of Sports Intelligence and Its Cooperation with Sports Science." This lays the foundation for the emergence of sports competitive intelligence in the future.

\subsection{The Applied Research of Sports Intelligence at this Stage}

The foreign sports intelligence work develops rapidly in this period, but generally speaking, it mainly focuses on the practical research, and does not pay enough attention to the theoretical research. Some scholars compared the whole sports intelligence work in this period into a triangle: literature is the basis of intelligence work, research is the central link or advanced form of intelligence work, and retrieval is the key to find literature and the ladder leading to research, and one of the three cannot be missing [6], as shown in figure 1 . The 
applied research of sports intelligence is also developed in accordance with the above model, which applies the "research" (research results, namely sports intelligence) obtained from the "document-retrieval-research" to practice. As the differences in sports training methods and means decrease, the one who can grasp the intelligence of the opponent more accurately and comprehensively in the international competitions will have a greater possibility to win. Therefore, the sports powers all put the sports intelligence in the important position, and edits and spreads the sports intelligence with the help of the technology means. For example, Japan sent more than 900 people to Rome to collect information on various national projects in preparation for the Tokyo Olympic Games. On the eve of the Chinese women's baseball team visit to the United States, the U.S. embassy in China invited the Chinese women's baseball team to have a friendly match with the embassy staff. The U.S. embassy made a full-court video. Once again, our country held a national youth table tennis competition in taiyuan, Japan table tennis association sent three cameramen from different angles to take pictures ofChinese reserve forces, even grip and racket are not missed. In the late 1970 s, for example, in order to change the long-term backward situation in the international volleyball world, the United States volleyball world has established a large and systematic information network. The United States men's and women's volleyball teams have both jumped into the world's leading ranks in a short time by collecting and paying attention to the technical and tactical developments of each great nation in volleyball, using computer and other equipment to analyze the data and develop corresponding strategies for targeted training.

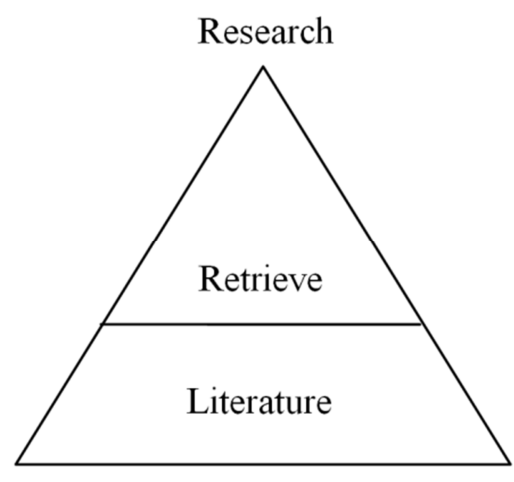

Figure 1. The Entire Intelligence Map.

To sum up, the main achievements and features of the traditional sports intelligence stage: 1) diversification of literature work; 2) computerization of retrieval, storage miniaturization; 3) deepening of international cooperation; 4) initialization of their own infrastructure. The international sports intelligence work is progressing rapidly, but its research mainly focuses on the development and use of literature retrieval system, literature quantification and automation construction and other literature management work, without paying attention to theoretical research. As a result, the distinction between sports intelligence work and traditional library science and literature work is not clear, and the boundary between intelligence science and library science is increasingly blurred.

\section{Modern Sports Intelligence Stage: The Rise of Sports Competitive Intelligence (From the End of 1990s to the Present)}

\subsection{The Rise of Sports Competitive Intelligence}

After more than 10 years of "information" and "intelligence" debate, the storm gradually subsided, the result is that intelligence science has become an independent sub-discipline of information science. Under the impact of the wave of network and knowledge, intelligence science should make corresponding changes in line with the trend of the times. So how to develop and position information science has become a key issue. Modern competitive intelligence emerged in the 1950 s and rose in the 1980s. It is a process in which people collect, analyze and disseminate accurate, timely and actionable information about the competitive environment, competitors and organizations themselves in a professional and ethical manner [7]. Competitive intelligence is an important development of intelligence science. Competitive intelligence is an important development of Information Science. At the same time, the emergence of competitive intelligence has also brought space for the development of sports intelligence. As a derivative of competitive intelligence and sports intelligence, sports competitive intelligence emerged in the late 1990s. Through continuous development, sports competitive intelligence work has become an indispensable part of competitive sports. In particular, with the advent of the era of big data, the application of big data technology has had a great impact on sports competitive intelligence analysis and promoted the in-depth development of sports competitive intelligence work.

\subsection{The Sports Competitive Intelligence Work Team}

If we talk about the relationship between professional sports and competitive intelligence, we have to start with American professional sports, not to mention that the professionalization of American sports is in the leading position in the world, only that the ancestor of professional sports is the national league of baseball founded in 1876. After 140 years of development, sports competitive intelligence has occupied a pivotal position in American professional sports. According to a survey, 62\% of the 135 professional league teams in MLB, NFL, NHL, NBA and MLS have competitive intelligence, 52\% have professional intelligence teams and $43 \%$ have their own intelligence teams. The team also purchases intelligence and data services [8]. In basketball, for example, each NBA team has a professional team, competitive intelligence main work content as follows: the use of high-tech means to match the scene real-time data, to set up the league players tactical database, database and team competition environment information collection, such as facilities, the referee information, climate condition, board and lodging and 
transportation, the atmosphere and its fans, etc.), for the coaches to provide competitive intelligence work which is in the face of the opponent's plan. The sports competitive intelligence achievement system presented by intelligence strategic value is shown in figure 2 .

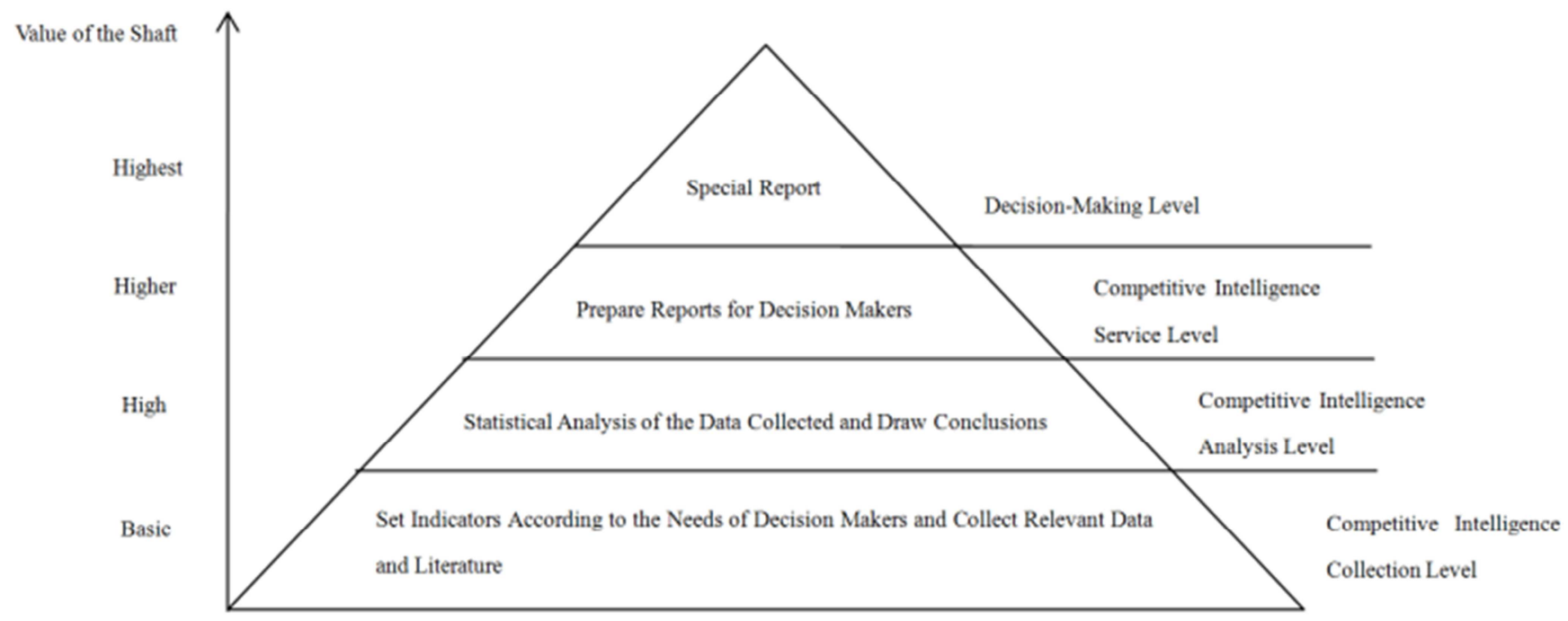

Figure 2. The Achievement System of Sports Competitive Intelligence.

In addition to the teams having their own competitive intelligence teams, there are many independent third-party researchers and institutions engaged in this research. For example, Dean Oliver, a prominent basketball data analyst, represents Basketball on Paper, whose research interests include the number of offensive-defensive transitions, the pace of the game, the impact of the team as a whole on personal data, and the importance of players' ability to create points. The four key elements of basketball success (shooting percentage, offensive rebound, free throw and mistakes) that he has proposed become an important basis for analyzing and evaluating players and teams [9]. John Hollinger, a famous basketball data analyst, founded the Air Relay website in 1996 , which mainly writes NBA reviews and analyzes NBA data. His book Pro Basketball Forecast/ Prospectus is a classic in the field of basketball statistical analysis. His original "PER" (Player Efficiency Rating), which is used to calculate the true contribution of a player in a match, is now widely used by teams and the media. Even many data websites use "PER" as a regular statistical indicator for players [10]. For another example, Synergy Sports Technology, founded in 2000, combined the detailed data that coaches want with video of each data, that is, each data has corresponding video for users to refer to, in the hope of becoming "Google in the field of basketball technical and tactical analysis."

\subsection{The Sports Competitive Intelligence Work Under High Technology}

With the progress of science and technology, more and more high-tech means are involved in the field of sports, so that sports competitive intelligence presents more and more high accuracy and instantaneity. Especially since the beginning of the 21 st century, the real-time data collection, statistics and analysis services under the high-tech competition scene have brought tremendous changes to competitive sports.
For example, major league baseball's statcast system provides real-time tracking and graphical data analysis services for users, such as when a player swings and hits the ball, which can map the ball's flight path, predicting its final landing point, and speed on a computer or television screen [11]. Any promising technology must be built on a foundation that is people-centered and helps improve the quality of human life, and the NBA has always been an outstanding representative of professional sports in terms of "making full use of technology to accelerate my own development." Here are a few NBA examples: 1) SportVU, developed in 2005 by Mickey Tamir, an Israeli missile-tracking and optics expert, is now used primarily in basketball programs. SportVU is like a tracking and analysis system for players. It is like a monitor, recording everything in the time of the game. It can quickly and accurately present the data of players' on-field efficiency, team's gain and loss in each round, etc [12]. 2) ShotTracker -- a wearable device to improve shooting technology. This device can calculate the shot accuracy and strength of the shooter by using the wrist protector, the chip in the elbow protector and the sensor in the supporting basket network. Users can query the shot data and analyze with the mobile APP, and then do targeted training. 3) The whistle of the NBA referee is equipped with multiple sensors, which can ensure that the timer will stop synchronously with the whistle. If a match is to be resumed, the referee will simply press the button on the device he is wearing and the timer will be restarted, thus ensuring that each second of the crucial moments of the match will be allocated fairly. 4) The $360^{\circ}$ angle of playback technology, FreeD, it is through the field more high-definition cameras to capture every angle special of image and image data summary, which finally provides users with a 3D perspective images. 5) There are other high-tech things that haven't been put into use, such as an Australian specialist who has developed an artificial eye that looks like a dragonfly's field of vision, a bionic tool that allows players to see $360^{\circ}$ without dead angles, allowing more point 
guards to pass the legendary "no-pass" ball. Sports medicine specialists, for example, have been able to repair players' damaged ligaments using nanotechnology such as artificial biomaterials and polyester, once the technology is available [13].

The magazine "Competitive Intelligence" hosted by the society of competitive intelligence professionals listed "data analysis as a competitive intelligence tool" as the fifth in a 2012 article titled "ten trends in competitive intelligence" [14]. It can also be seen from the above examples that data is an effective tool for competitive intelligence, and high technology has brought tremendous changes to the collection and analysis of data. More and more teams are able to accept the training, competition and management of teams enriched by scientific means.

\subsection{The Main Sports Item of Sports Competitive Intelligence Research}

In addition to the United States, sports competitive intelligence also affects sports around the world. In this paper,
3,973 papers on sports competitive intelligence were retrieved from the Scopus database, and classified according to different sports items, and the top 30 popular sports items in the sports competitive intelligence field in figure 3 were obtained. Among them, football ranked first with the number of published papers of 722 and the number of cited papers of 18,560. Among the hold rackets, net screens, and counter items, tennis received the highest attention, with 90 articles, ranking No.10. In the fighting confrontation items, the hottest one is judo, a total of 44 articles, ranking 18 th. It can be seen from figure 3 that almost all the international mainstream sports items are within the scope of research in the field of sports competitive intelligence. It can also be found that only skating and skiing are winter Olympic sports, and both of them are not ideal, ranking 16th and 20th respectively, showing that winter sports are relatively lacking in the study of sports competition intelligence.

\section{Items}

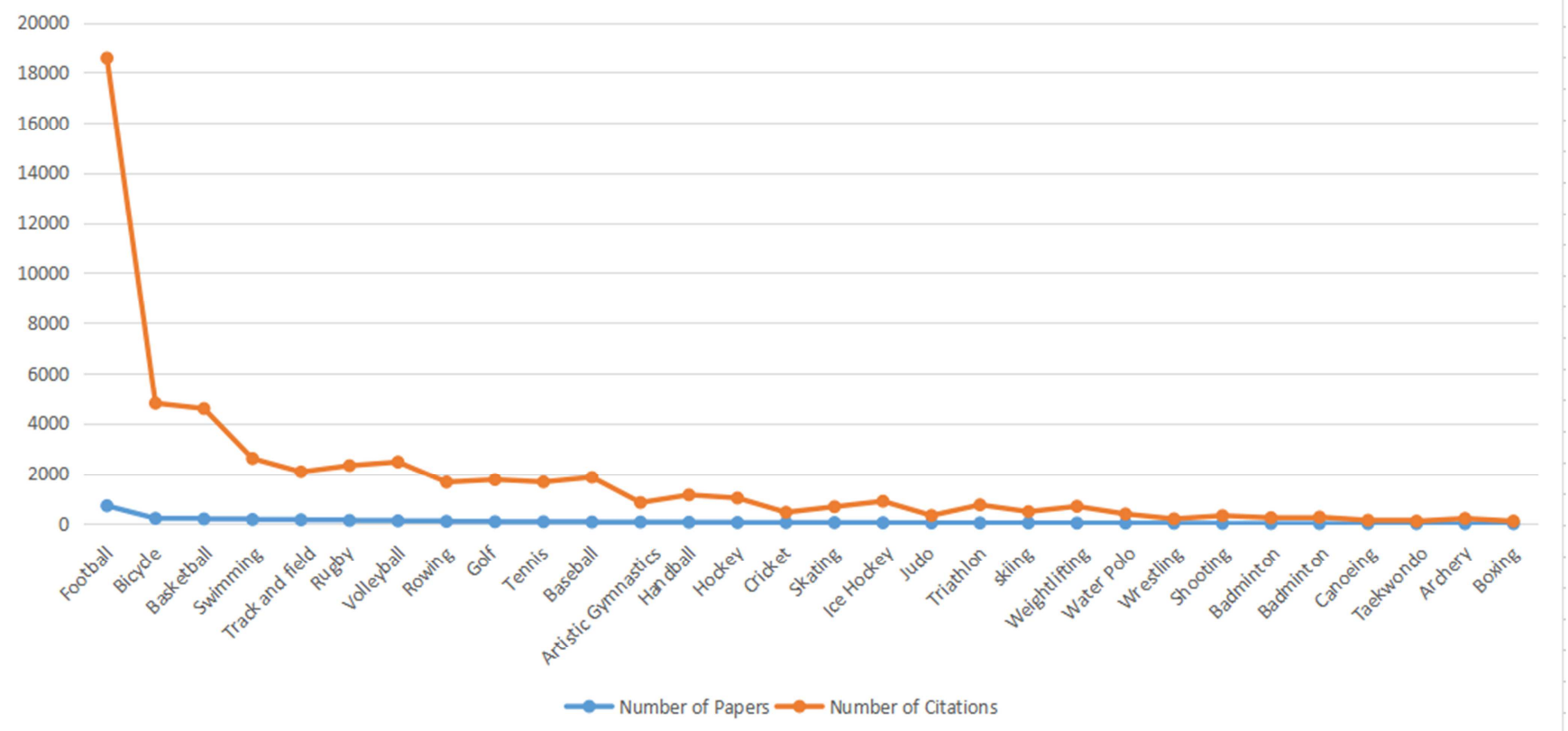

Figure 3. The Top Sports in Sports Competitive Intelligence.

To sum up, the main achievements and characteristics of modern sports intelligence stage are as follows: 1) Sports competitive intelligence began in the late 90s and developed rapidly and smoothly in the 21 st century. Its remarkable feature is that the intelligence work has changed from sports literature processing to real-time data collection and statistics by using high-tech means. And analytical services have brought great changes to competitive sports. 2) In addition to the teams having their own competitive intelligence teams, many independent third-party researchers and institutions are also engaged in this research. 3) With the explosive growth of the amount and types of data available for competitive intelligence work, users can put forward various requirements to competitive intelligence providers according to their own needs. The so-called "customized service" of intelligence has become a reality. 4) Most of the international mainstream sports are within the scope of sports competitive intelligence research; winter sports are relatively lack of sports competitive intelligence research.

\section{Concluding Remarks}

Sports competitive intelligence has experienced three important stages: the pioneer stage, the traditional sports intelligence stage and the modern sports intelligence stage. The future development of sports competitive intelligence will be accompanied by the rapid development of modern information technology such as Internet, big data, cloud computing, and economic, political, cultural, social and other aspects of change showing the trend of diversification of 
service objects, methods and tools of science and technology, research paradigm diversification. The development prospects of sports competitive intelligence and its enlightenment to China are as follows: 1) Data collection is one of the key elements of sports competitive intelligence. The continuous development of science and technology will bring more high-tech data acquisition software and equipment to this field. At present, the widely used GPS player positioning software, Israe's SportVU player tracking analysis system, Australia's Gamebreaker game analysis software, Switzerland's Dartfish game analysis software and player management software Cronus. In the near future, the upgrading of these technologies and the introduction of new technologies will bring greater room for development in this field. 2) With data, accurate and effective data analysis is needed. The new multivariate statistical model will make the sports competitive intelligence work more forward-looking and predictive, so as to provide coaches with more accurate, effective and timely competitive intelligence services, such as mixed linear model, multivariate logarithmic regression model, neural network model has been gradually introduced into the sports competitive intelligence work. Come on. 3) Sports competitive intelligence workers in China should grasp the changing trend of competition in time and explore ways to quickly extract, correlate and reorganize relevant data from mass data so as to provide users at all levels with efficient and high-quality solutions to meet their decision-making needs.

\section{References}

[1] Sports information theory and practice research group. Theory and practice of sports information work [M]. Beijing: People's Physical Culture Publishing House, 1988: 4.

[2] Ma, T. Academic and work trends of the eighth international sports information conference $[J]$. Sports Science, 1986, 6 (2): 76-78.

[3] Liu, C. Study on the sports competitive intelligence and its influence athletics sports core competence [D]. Shanghai: Shanghai University of Sports, 2010.

[4] Fearing Boff. Several theoretical problems of information assurance in sports field [J]. Journal of Wuhan Sports University, 1988, 4(3): 5-7.

[5] Nye Bono. Problems and prospects of sports informatization [J]. Sports Science and Technology Information, 1995, 15(2): 1-2.

[6] Hughes, M. D. \&Evans, S. Establishing normative profiles in performance analysis [J]. International Journal of Performance Analysis in Sport, 2001, 1(1): 1-26.

[7] Bergeron, P. \&Hiller C. A. Competitive intelligence [J]. Annual Review of Information Science and Technology, 2002, 36(1): 353-390.

[8] Morran, C. HBO Seeks Streaming Video Help From Major League Baseball [J/OL].

[2014-12-09].http://consumerist.com/2014/12/09/hbo-seeks-st reaming-video-help-from-majorleague-baseball.

[9] Oliver, D. Basketball on paper [M]. USA: Potomac Books Press, 2004: 29-31.

[10] Hollinger, J. Pro basketball forecast/prospectus [M]. USA: Potomac Books Press, 2005: 26-28.

[11] Lewis, M. Moneyball: The art of winning an unfair game [M]. New York: W. W. Norton \& Company, 2013: 45-46.

[12] Tami, M. Real-time objects tracking and motion capture in sports events [P]. US Patent App: 11/909, 080, 20065.

[13] Reilly, T. Assessment of sports performance with particular reference to field games [J]. European Journal of Sport Science, 2001, (1): 12 .

[14] Liao, Q. Data intelligence analysis behind "Crazy Lin" [J]. Competitive Intelligence, 2012, (1): 1. 